\title{
Parkinsonian signs and symptoms in adults with a history of Sydenham's chorea
}

\author{
Leonardo Brandão Barreto, Ricardo Oliveira Horta Maciel, Débora P. Maia, Antonio Lúcio Teixeira, \\ Francisco Cardoso*
}

Movement Disorders Clinic, Neurology Service, Department of internal Medicine, Universidade Federal de Minas Gerais, Av Pasteur 89/1107, 30150-290 Belo Horizonte MG, Brazil

\section{A R T I C L E I N F O}

\section{Article history:}

Received 6 September 2011

Received in revised form 26 October 2011

Accepted 1 November 2011

\section{Keywords:}

Sydenham's chorea

Chorea

Parkinsonism

Bradykinesia

\begin{abstract}
A B S T R A C T
Background: Sydenham's chorea is associated with dysfunction of fronto-striatal circuits induced by crossreactive antibodies to group A $\beta$-hemolytic streptococcus. High susceptibility of extrapyramidal effects of neuroleptics in patients with Sydenham's chorea suggests underlying nigro-striatal dysfunction. Objective: To study the presence of parkinsonism in patients with a history of Sydenham's Chorea. Methods: We used the UFMG Sydenham's Chorea Rating Scale (USCRS) and the Unified Parkinson's Disease Rating Scale (UPDRS) part III, respectively, to determine the presence of chorea and parkinsonian symptoms and signs in 25 adults with a history of previous Sydenham's Chorea currently without chorea or use of anti-choreic drugs.

Results: Bradykinesia was found in $64 \%$ of subjects. There was a statistically significant correlation between bradykinesia and hemichorea $(-0.412 ; p=0.036)$ and bradykinesia and generalized chorea (0.412; $p=0.036)$. There was no correlation between bradykinesia and use of anti-choreic drugs.

Conclusions: Bradykinesia is common in patients with Sydenham's Chorea in remission. This finding suggests an immune-mediated dysfunction of the nigro-striatal system.
\end{abstract}

(C) 2011 Elsevier Ltd. All rights reserved.

\section{Introduction}

Sydenham's chorea (SC) is one of the manifestations of rheumatic fever, an auto-immune disease related to previous infection with group A $\beta$-hemolytic Streptoccocus. Chorea is the cardinal motor feature of the illness, but other motor abnormalities include decreased muscle tone, tics, dysarthria, ocular motricity abnormalities and motor impersistency [1]. Recently, a wide spectrum of cognitive and behavioral symptoms such as attention deficit hyperactivity, emotional lability and obsessive-compulsive behavior have been recognized as part of SC [2,3].

SC is thought to be related to dysfunction of fronto-striatal circuits caused by cross-reactive antibodies targeting streptoccocal antigens_ENREF_9. Studies have demonstrated increased volume and hyperperfusion [4] of basal ganglia structures during acute SC. Indeed, anti-basal ganglia antibodies that target caudate, putaminal, and subthalamic cytoplasmatic proteins are present in up to $90 \%$ of SC patients $[5,6]$.

We have reported that in comparison with patients with Tourette's syndrome, subjects with SC have increased risk of developing parkinsonism and other extra-pyramidal side-effects when

\footnotetext{
* Corresponding author. Tel.: +55 31 32131055; fax: +55 3132134951.

E-mail address: cardosofe@terra.com.br (F. Cardoso).
}

exposed to neuroleptics [7]. There is also one case report describing the occurrence of parkinsonism in a patient with SC not treated with antidopaminergic agents [8]. One can speculate that during the early phase of disease exuberant chorea could mask subtle parkinsonian findings, which would surface in the latter stages of the disease, particularly after chorea has subsided. We found, however, no studies in the literature prospectively investigating the occurrence of parkinsonian signs and symptoms in SC. The occurrence of auto-antibodies targeting different regions of the basal ganglia circuitry in SC, including the nigro-striatal system [5], suggests that parkinsonism in these patients could be related to autoimmune-mediated striatal dysfunction.

The aim of this study is to evaluate the presence of parkinsonian signs and symptoms in adult patients with a history of SC.

\section{Patients and methods}

We prospectively examined 25 patients from our Movement Disorders Clinic from March 2006 to August 2007. Patients were 16 years or older, with previous history of SC and in the remission phase of chorea. Remission was defined as absence of chorea while off anti-choreic medications. Patients were excluded if being treated with medications deemed capable of inducing parkinsonism or presenting signs and symptoms of active SC. SC was diagnosed if patients met the modified Jones criteria for Rheumatic Fever and other causes of chorea ruled out.

A neurologist specialist in movement disorders (LB) examined patients for parkinsonian signs. Parkinsonism was defined as the presence of bradykinesia along with either rigidity, rest tremor or postural instability, according to the Step 1 of the 
Table 1

Clinical and demographic features of studied population.

\begin{tabular}{ll}
\hline$N$ & 25 \\
Male (\%)/Female (\%) & $8(32 \%) / 17(68 \%)$ \\
Age & 19.9 anos $( \pm 5.4)$ \\
Generalized chorea & $6(24 \%)$ \\
Hemichorea & $19(76 \%)$ \\
Carditis & $13(61.9 \%)$ \\
Arthritis & $6(28.5 \%)$ \\
Psychiatric abnormalities & $5(23.8 \%)$ \\
Use of Medications & $23(9 \%)$ \\
Time off medications for SC & 44 months $( \pm 32.8) ;$ minimum: \\
& 3 months; maximum: 100 months \\
USCRS score & $1.84( \pm 2.3)$ \\
UPDRS score & $1.16( \pm 1.08)$ \\
Bradykinesia & $16(64 \%)$ \\
\hline
\end{tabular}

Brain Bank Clinical Diagnosis Criteria for Parkinson's Disease. Patients were also carefully examined for the presence or other movement disorders which could interfere with the motor tasks, such as dystonia and tremor. The Unified Parkinson's Disease Rating Scale (UPDRS) part III and the Federal University of Minas Gerais Sydenham's chorea rating scale(USCRS) [9] were applied in the same visit. Only patients with a score of zero in the chorea item of the USCRS were included in this study.

We reviewed patients charts and recorded the type of movement disorder during the acute phase of SC (e.g. hemichorea or generalized chorea), presence of arthritis, carditis and behavioral abnormalities, use of anti-choreic medication, and time elapsed since off anti-choreic medications.

Statistical analysis was performed with SPPSS ${ }^{\circledR}$ version 13.0. Spearman test was used to evaluate the correlation between variables, with significance set at $5 \%$.

This study was approved by the local ethics comitee and all subjects signed a written informed consent.

\section{Results}

Table 1 shows clinical and demographic features of the studied population. Because of the importance of exposure to medications to interpret our data on parkinsonian findings, we carefully recorded the treatments used. Only two patients had never received drug treatment for SC. Ten were treated with neuroleptics (six received haloperidol, three pimozide, and one risperidone) and 13 were treated with valproic acid. Clonazepam and fluoxetin were used in one patient each. Of all patients, eight only used valproic acid, three only haloperidol, one only fluoxetin, two only pimozide and one only risperidone during the course of follow-up. Five patients had a regimen of more than one drug: two used valproic acid followed by haloperidol; one pizotifen, followed by valproic acid and then a tryciclic antidepressant; one with pimozide followed by valproic acid; and one with haloperidol, then valproic acid and later clonazepam.

Isolated bradykinesia was found in 16 (64\%) patients. However, in the absence of other cardinal features, including rigidity, parkinsonism was not diagnosed in any of the subjects. No correlation was found between bradykinesia and use $(p=0.052)$ or time of withdrawal ( $p=0.876)$ of antichoreic medications, none also in relation to age $(p=0.55)$ or gender $(p=0.45)$. Moreover, both of our untreated patients exhibited bradykinesia on motor examination, despite never being exposed to neuroleptics or other medications deemed capable of inducing parkinsonism. All patients with a previous history of generalized chorea had bradykinesia, a statistically significant finding $(p=0.036)$. Interestingly, a negative correlation was found between the history of hemichorea and presence of bradykinesia ( $p=0.036)$. Females were statistically more prone to having psychiatric abnormalities $(p=0.047)$. No other correlation was found with the presence of psychiatric symptoms, including bradykinesia, hemi- or generalized chorea.

Patients with bradykinesia where also assessed for the presence of limb dystonia which could interfere with the motor tasks used to rate bradykinesia. However, no dystonic posturing or tremor were observed in any of the patients during examination. Table 2 summarizes the results of the stastistical analysis.

\section{Discussion}

Overall, the demographic and clinical features of our population are consistent with previous reports of SC [10]. Over two thirds

Table 2

Correlation between studied variables.

\begin{tabular}{|c|c|c|c|c|c|c|c|c|c|c|}
\hline Variable & Statistics & Age & Sex & ToM & Bradykinesia & GC & $\mathrm{HC}$ & UoM & Arthritis & Carditis \\
\hline \multirow[t]{2}{*}{ Sex } & $\begin{array}{l}\mathrm{CC} \\
\text { ( } p \text { value) }\end{array}$ & $\begin{array}{c}-0.501 \\
0.001^{\mathrm{a}}\end{array}$ & & & & & & & & \\
\hline & $n$ & 25 & & & & & & & & \\
\hline \multirow[t]{3}{*}{ ToM } & $\mathrm{CC}$ & 0.340 & 0.062 & & & & & & & \\
\hline & (P) & 0.113 & 0.779 & & & & & & & \\
\hline & $n$ & 23 & 23 & & & & & & & \\
\hline \multirow[t]{3}{*}{ Bradykinesia } & $\mathrm{CC}$ & 0.125 & 0.157 & 0.034 & & & & & & \\
\hline & ( $p$ value) & 0.553 & 0.453 & 0.876 & & & & & & \\
\hline & $n$ & 25 & 25 & 23 & & & & & & \\
\hline \multirow[t]{3}{*}{ GC } & $\mathrm{CC}$ & 0.147 & 0.217 & 0.105 & 0.421 & & & & & \\
\hline & ( $p$ value) & 0.484 & 0.298 & 0.635 & $0.036^{\mathrm{a}}$ & & & & & \\
\hline & $n$ & 25 & 25 & 23 & 25 & & & & & \\
\hline \multirow[t]{3}{*}{$\mathrm{HC}$} & $\mathrm{CC}$ & -0.147 & -0.217 & 0.105 & -0.421 & -1.000 & & & & \\
\hline & ( $p$ value) & 0.484 & 0.298 & 0.635 & $0.036^{\mathrm{a}}$ & $<0.001^{\mathrm{a}}$ & & & & \\
\hline & $n$ & 25 & 25 & 23 & 25 & 25 & & & & \\
\hline \multirow[t]{3}{*}{ UoM } & $\mathrm{CC}$ & 0.042 & 0.202 & -0.273 & 0.393 & 0.166 & 0.166 & & & \\
\hline & ( $p$ value) & 0.842 & 0.332 & 0.207 & 0.052 & 0.429 & 0.429 & & & \\
\hline & $n$ & 25 & 25 & 23 & 25 & 25 & 25 & & & \\
\hline \multirow[t]{3}{*}{ Arthritis } & $\mathrm{CC}$ & 0.062 & 0.155 & 0.098 & 0.062 & 0.141 & 0.141 & 0.205 & & \\
\hline & ( $p$ value) & 0.788 & 0.502 & 0.689 & 0.789 & 0.541 & 0.541 & 0.372 & & \\
\hline & $n$ & 21 & 21 & 19 & 21 & 21 & 21 & 21 & & \\
\hline \multirow[t]{3}{*}{ Carditis } & $\mathrm{CC}$ & 0.050 & 0.192 & 0.229 & 0.192 & -0.022 & 0.022 & 0.080 & -0.155 & \\
\hline & ( $p$ value) & 0.830 & 0.404 & 0.345 & 0.404 & 0.925 & 0.925 & 0.732 & 0.502 & \\
\hline & $n$ & 21 & 21 & 19 & 21 & 21 & 21 & 21 & 21 & \\
\hline \multirow[t]{3}{*}{ Psychiatric abnormalities } & $\mathrm{CC}$ & 0.388 & -0.439 & -0.033 & -0.252 & -0.313 & 0.313 & -0.200 & -0.354 & -0.022 \\
\hline & ( $p$ value) & 0.082 & $0.047^{\mathrm{a}}$ & 0.894 & 0.270 & 0.168 & 0.168 & 0.386 & 0.116 & 0.925 \\
\hline & $n$ & 21 & 21 & 19 & 21 & 21 & 21 & 21 & 21 & 21 \\
\hline
\end{tabular}

Legends to Table 2: CC - correlation coeficient; GC - generalized chorea; HC - hemichorea; ToM - time off medications; UoM - use of medications.

a Stastisticaly significant correlation. 
(68\%) of our patients were female. A history of carditis was present in $13(61.9 \%)$ patients and arthritis in $6(28.5 \%)$. There are, however, a few discrepancies. Hemichorea was present in $76 \%$ of our patients, whereas $24 \%$ had generalized chorea. Our study had a higher proportion of patients with hemichorea than previously reported [1]. This could be due to selection bias or possibly that hemichorea is a less severe form of chorea with a higher chance of remission. When compared to patients with other neurologic conditions or normal individuals, regardless of gender, patients with a history of SC have a higher frequency of psychiatric disease often with a chronic course and difficult management [11]. We observed behavioral abnormalities in 5 of our patients (20\%), a small frequency when compared to other studies [2]. This is most probably due to detection of psychiatric features not being the primary objective of the study, which was based on chart review, without the use of more sensitive and reliable scales that could improve detection of psychiatric symptoms.

Our study found a high prevalence of $64 \%$ of bradykinesia in patients with a history of SC. One question is whether we are dealing with true bradykinesia or poor performance on the bradykinesia items of the UPDRS scale as a result of presence of residual chorea. To avoid this problem, we excluded patients with persistent chorea from the study. Moreover, the fact that these patients also had a total score on the USCRS lower than 2 points, with the chorea items being 0 by inclusion criteria, strongly suggests that the patients had true bradykinesia, and not motor incoordination from subtle persistent chorea. The absence of dystonic posturing also rules out dystonia of the limbs as a confounder in this study. Patients therefore were considered to have true bradykinesia, defined in the UPDRS as slowness with fatigability or hesitation. Despite, however, of the high frequency of bradykinesia, no other cardinal feature of parkinsonism was detected in any of the patients. Bradykinesia did not correlate with age, gender, psychiatric symptoms, carditis or arthritis. On the other hand, bradykinesia was more frequent in patents with a history of generalized chorea, present in all of the six patients with this presentation of chorea in our sample. Interestingly, there was a negative correlation between bradykinesia and a history of hemichorea, which was also statistically significant. It could be that generalized chorea, being a marker of more severe and widespread damage of basal ganglia circuitry during the acute phase of the disease, is associated with some degree of nigrostriatal dysfunction, thus making the presence of bradykinesia a late marker of disease severity after remission of chorea in SC patients.

A significant proportion of patients were treated during the acute phase of disease with neuroleptics and valproic acid, both of which can cause drug-induced parkinsonism [12]. Patients with SC appear to have a greater sensitivity to neuroleptics than agematched subjects with TS treated with the same chlorpomazineequivalent doses. Extrapyramidal side-effects occur not only more frequently but also with smaller doses [7]. In this study, however, previous use of antichoreic medications (including neuroleptics) as well as the time elapsed since off the medication and clinical evaluation did not correlate with the presence of bradykinesia. Median time between last dose of medication and study examination was 44 months, and the presence of bradykinesia in drugnaïve patients point to the conclusion that SC is an independent risk factor for the occurrence of bradykinesia, regardless of previous use of antidopaminergic agents. In this regard, the accepted criteria for drug-induced parkinsonism establishes the maximum time frame of 6 months after use of the offending drug in order to establish a causal link between the two [12]. We thus believe that the pathogenesis of bradykinesia in patients with SC in remission might be related to an underlying immune-mediated dysfunction of the nigro-striatal system. Interestingly, the progression from one end of the spectrum of disorders of movement, hyperkinesia, to the opposite end, bradykinesia, is somewhat reminiscent of the clinical picture typically seen in patients with Huntington's disease, albeit in a much less severe way. The systematic use of dopaminergic imaging is warranted to further investigate the role of nigro-striatal dysfunction in the pathogenesis of bradykinesia in SC patients.

In conclusion, to our knowledge, this is the first study to prospectively investigate the presence of parkinsonian signs in a consecutive sample of SC patients. We were able to identify bradykinesia in $64 \%$ of our cohort. The finding that bradykinesia correlated with the more severe form of chorea in the acute phase of SC, and not with previous use of antichoreic drugs, suggests that there is an immuno-mediated dysfunction of the nigro-striatal system in this condition.

\section{Conflict of interest}

Leonardo Brandão Barreto has no conflict of interest to declare Ricardo Olveira Horta Maciel has no conflict of interest to declare Débora Palma Maia has no conflict of interest to declare Antonio Lúcio Teixeira Jr. has received research grants from $\mathrm{CNPq}$ and FAPEMIG. Francisco Cardoso has received honoraria from Boehringer-Ingelheim, Novartis and Roche; and research grant from FAPEMIG.

\section{Authors roles}

1 Research project: A. Conception, B. Organization, C. Execution;

2 Statistical Analysis: A. Design, B. Execution, C. Review and Critique;

3 Manuscript Preparation: A. Writing of the first draft, B. Review and Critique;

Leonardo Brandão Barreto - 1B, 1C, 2A, 2B, 2C, 3A, 3B Ricardo Oliveira Horta Maciel - 1C, 3A Débora Palma Maia - 1C Antônio Lúcio Teixeira Jr. - 1C Francisco Cardoso - 1A, 1B, 1C, 2A, 2C, 3A, 3B.

\section{References}

[1] Cardoso F, Eduardo C, Silva AP, Mota CC. Chorea in fifty consecutive patients with rheumatic fever. Mov Disord 1997;12:701-3.

[2] Maia DP, Teixeira AL, Quintão Cunningham MC, Cardoso F. Obsessive compulsive behavior, hyperactivity, and attention deficit disorder in Sydenham chorea. Neurology 2005;64:1799-801.

[3] Teixeira AL, Athayde GR, Sacramento DR, Maia DP, Cardoso F. Depressive and anxiety symptoms in Sydenham's chorea. Mov Disord 2007;22:905-6.

[4] Barsottini OG, Ferraz HB, Seviliano MM, Barbieri A. Brain SPECT imaging in Sydenham's chorea. Braz J Med Biol Res 2002;35:431-6.

[5] Husby G, van de Rijn I, Zabriskie JB, Abdin ZH, Williams Jr RC. Antibodies reacting with cytoplasm of subthalamic and caudate nuclei neurons in chorea and acute rheumatic fever. J Exp Med 1976;144:1094-110.

[6] Church AJ, Cardoso F, Dale RC, Lees AJ, Thompson EJ, Giovannoni G. Anti-basal ganglia antibodies in acute and persistent Sydenham's chorea. Neurology 2002;59:227-31.

[7] Teixeira AL, Cardoso F, Maia DP, Cunningham MC. Sydenham's chorea may be a risk factor for drug induced parkinsonism. J Neurol Neurosurg Psychiatry 2003; 74:1350-1.

[8] Ben-Pazi H, Livne A, Shapira Y, Dale RC. Parkinsonian features after streptococcal pharyngitis. J Pediatrics 2003;143:267-9.

[9] Teixeira AL, Maia DP, Cardoso F. UFMG Sydenham's chorea rating scale (USCRS): reliability and consistency. Move Disord 2005;20:585-91.

[10] Goldenberg J, Ferraz MB, Fonseca AS, Hilario MO, Bastos W, Sachetti S. Sydenham chorea: clinical and laboratory findings. analysis of 187 cases. Rev Paul Med 1992;110:152-7.

[11] Swedo SE, Leonard HL, Schapiro MB, Casey BJ, Mannheim GB, Lenane MC, et al. Sydenham's chorea: physical and psychological symptoms of St Vitus dance. Pediatrics 1993;91:706-13.

[12] Barbosa MT, Caramelli P, Maia DP, Cunningham MC, Guerra HL, LimaCosta MF, et al. Parkinsonism and Parkinson's disease in the elderly: a community-based survey in Brazil (the Bambui study). Mov Disord 2006;21: $800-8$. 